\title{
An Improved Analysis of Mac IEEE 802.16 in Wireless Ad Hoc Networks
}

\author{
R. Manikandan \\ Assistant Professor, \\ Dept. of Computer Science \& Engineering \\ Annamalai University \\ Chidambaram.
}

\author{
K. Selvakumar, PhD. \\ Associate Professor \\ Dept. of Computer Science \& Engineering \\ Annamalai University, \\ Chidambaram.
}

\begin{abstract}
In Wireless Ad Hoc Networks, the delay performance in data transmission reduces the rate of transmission in Mac layer, which affects the throughput in network. By comparing with existing work and proposed Mac protocol IEEE 802.16, This study carries out a MAC analysis that is of particular focus on networks which is deployed to provide $k$-coverage for real-time applications, to improve the Uplink and downlink Ratio in rated transmission. It is shown that introducing sensing for lower densities (i.e., in sparse networks) is not beneficial, while for higher densities (i.e., in dense networks), using an optimized sensing threshold provides significant gain. This simulate in ns2 with different topology to define the Mac issues.
\end{abstract}

Keywords: Wireless Ad Hoc Networks topology, Distributed Mac Protocols, Mac Issues, Adaptive configuration Algorithm, performance evaluation.

\section{INTRODUCTION}

Wireless Ad Hoc Networks are often used for real-time applications, such as environment surveillance, medical care, and vehicle traffic control. In these contexts, in spite of the severe resource limitations that characterize sensor nodes, MANET have to provide a reliable coverage of the area of interest as well as to meet severe timing constraints. Using an appropriate medium access control (MAC) protocol is one such technique. Taking into account the system's quality of service (QoS) requirements, a MAC protocol for ad hoc networks shares the medium and the available resources in a distributed manner, and allows for efficient interference management.

This paper considers different topologies in which nodes are randomly distributed in space, and addresses the problems occuring in data transmission through MAC layer design. The ALOHA and CSMA are the MAC protocols which are used to increase the packet delivery rate. In particular, the transmission rate can be analysed by having a fixed threshold on each transmitter (Tx) and receiver (Rx), and using MAC protocol of IEEE 802.16 the performance can be improved. IEEE 802.15 (Low Rate) deals with low data rate but very long battery life (virtual energy) and very simple. Power is derived from external sources in network. Unicast routing is the process of forwarding unicasted traffic from a source to a destination on an internetwork. Unicasted traffic is destined for a unique address. The related survey on Mac issues is as follows:

\subsection{CSMA/CD (carrier sense multiple access/ collision detection)}

Every node senses the carrier before transmitting. If the carrier is not clear, the node defers transmission for a specified period. Otherwise, transmits [1]. While transmitting, the sender is listening to carrier and sender stops transmitting if collision has been detected.

\subsection{Packet delay}

Packet delay results are presented as a function of a number of stations and packet size for basic access and RTS=CTS medium access mechanisms. Packet measurements need to span over a long period of time[1]. Queuing delay introduces a variable component to the point-to-point delays experienced by packets, as it depends on the traffic load along the path, which varies at different links and with time.

\subsection{Routing Misbehavior}

Routing information is needed to keep track of route changes, if any. Other supplementary data, such as fiber maps and router configuration information, are needed to address path-specific concerns.

A network is considered in which packets are located randomly in space and time according to a position initially, The packets, which are assumed to be of constant length, are forwarded by each TX over a non-fading channel to an RX a fixed distance away[2]. Wireless medium is an open, shared, and broadcast medium, and multiple nodes may access the medium at the same time. Predefined traffic management rule is the main advantage in the proposed protocol.[1] the analysis identifies when the RTS=CTS mechanism achieves lower packet delay with respect to the basic access mechanism and becomes highly beneficial for the performance of IEEE 802.11, The Collision avoidance mechanisms are (a), Collision avoidance with out-of-band signaling and (b), Collision avoidance with in-band control messages. By applying the energy throughout the network, The usage of energy in the entire network could be found.

\section{Adaptive configuration algorithm}

If a node has insufficient energy or moves frequently then it is not able to transmit the packet to the destination. This study defines a new rule which will show the trust on node while transmission, but source and the destination have peak energy when comparing with other nodes in the network [5]. Thus the parameter is as follows 


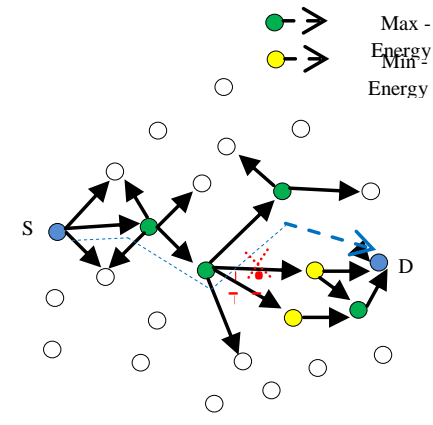

Fig 1: Routing Scheme with Energy Selection Individual Energy,

IE = We, n (1 - (Energy / Energy Full), + n Ws (Mobility / Mobility max)

Equation (1)

Where,

$$
\begin{aligned}
& \mathrm{N}=\text { node } \\
& \text { Energy = residual energy } \\
& \text { Mobility = current mobility } \\
& \text { Energy Full=full energy } \\
& \text { Mobility max= Max mobility level }
\end{aligned}
$$

Mobility level by two weights;

$$
\begin{aligned}
& \mathrm{W}_{\mathrm{e}, \mathrm{n}}=1-(\text { Energy/Energy Full }) \\
& \mathrm{W}_{\mathrm{s}, \mathrm{u}}=\left(1-\mathrm{W}_{\mathrm{e}, \mathrm{u}}\right)
\end{aligned}
$$

This adaption rule can improve the life time of node in the network, given in fig1. If a node has insufficient energy or moves frequently, a smaller that leads to a lower node degree, transmission radius, and power is desired.

\subsection{HIGH MOBILITY VS. LOW MOBILITY}

If a node moves frequently, its links are unstable, which in turn costs more energy for route reconstruction, and deteriorates the quality of the established routes. In this case, the node should keep a lower degree to reduce its dependency on nearby nodes by turning up [5] .On the contrary, if a node has lower mobility, it should turn down its to construct more reliable routes. The energy efficiency of routes, and a node u can conserve its own energy by simply brocasting to hop nodes.

\section{PROPOSED APPROACH}

The overall system has two modules:

3.a. Design of wireless network topology

3.b. IEEE- 802.16 Mac Protocol

Design of wireless network topology: Different topology is considered to compare their working performance on data transmission such as end to end delay, throughput, and packet delivery ratio and network lifetime improvement in network. Wireless communication results in power consumption, and migrates a task from an energy-constrained mobile host to an AC-powered base station. The previous works do not consider any task timing constraints, discuss how to combine remote processing and power management techniques to achieve further energy saving. The present work targets a mobile device providing real time services in a client-server wireless network. The objective is to minimize power consumption of the wireless nodes by using Mac protocol and dynamic power management while meeting some realtime constraints.

Possible factors which can induce benign dropping

include the following:

- mobility of nodes,

- network/traffic density,

- $\quad$ traffic type, and

- Channel and fading conditions.

Mobility makes the routes dynamic, i.e., an active route can become broken due to mobility. Here, the dropping of the packets becomes inevitable, as re-establishing a new route takes some time. Furthermore, mobility creates changing channel and fading conditions. Dropping can also be due to signal loss, interference, etc. Network and traffic density are crucial factors. The type of traffic characterizes the randomness in interval between transmissions and packet size. For example, a video streaming has a constant bit rate (CBR) with a fixed bandwidth

Bandwidth fixed in the Mac layer for video transmission is

$$
\begin{aligned}
& \text { BW }=100, \\
& \text { new_flow }=30 ; \\
& \text { th_Bw }=(85 / 100) * B w ; \\
& \text { Bw_realtime= }(80 / 100) * \text { th_Bw; } \\
& \text { real_datarate=8; } \\
& \text { bwavg1=35; } \\
& \text { bwavg2=30; } \\
& \text { bwavg3=45; }
\end{aligned}
$$

Transmissio1: Bwflow1=bwavg1+new_flow;

Transmissio2: Bwflow2=bwavg2+new_flow;

Transmission3:Bwflow3=bwavg3+new_flow;

In Mac layer bandwidth allocation will be dynamic or constantly specified above. The distance between two fixed nodes is set to 390 meters, which was selected to be able to setup connections for all single rate MAC protocols. Since the receiving power sensitivity gets higher as the capacity of the modulation increases, This study picks a distance smaller and close to the shortest sensitivity range as the simulated distance, which is from $11 \mathrm{Mbps}$ single rate protocol. 


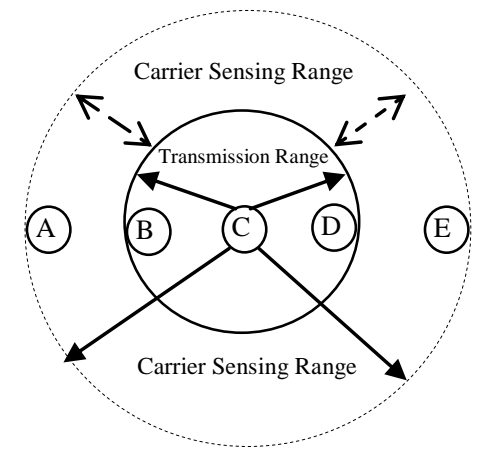

Fig 2: Nodes in transmission range in the carrier sensing zone

As the receiving power changes, each RTS/CTS exchange will cause a channel capacity re-negotiation. The CBR throughput of BRAR with single rate MAC protocols are $1 \mathrm{Mbps}, 2 \mathrm{Mbps}, 5.5 \mathrm{Mbps}$ and $11 \mathrm{Mbps}$. The throughput is the average CBR throughput in 1 second time period. As expected, the rate-adaptive MAC protocol based on the Receiver-Based Auto-Rate gets the highest throughput in a fading channel. However, the $11 \mathrm{Mbps}$ MAC protocol performs poorer than expected. It could be caused by the distance of 390 meters, which is close to the max sensitivity range of the 11 Mbps MAC protocol and fading may cause greater effects than in other cases.

\subsection{TRANSMISSION RANGE}

When a node is within transmission range of a sender node, it can receive and correctly decode packets from the sender node. In the present simulations, the transmission range is $250 \mathrm{~m}$ when using the highest transmits power level.

Carrier sensing zone: When a node is within the carrier sensing zone, as per the definition here, the carrier sensing zone does not include transmission range. Nodes in the transmission range can indeed sense the transmission, but they can also decode it correctly. Therefore, these nodes will not be in the carrier sensing zone as per the definition. The carrier sensing zone is between $250 \mathrm{~m}$ and $550 \mathrm{~m}$ with the highest power level in simulation.

\subsection{POWER LAW}

Without loss of generality, we assume a common channel model that follows the power law

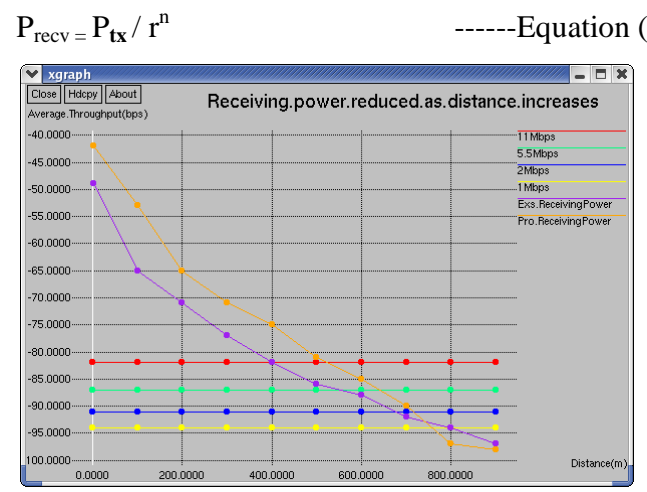

Fig 3: Comparison of Receiving Power Adaption
Where Precv is the strength (or power) of the signal when it arrives at a receiver, $P t x$ is the transmission power, $r$ is the transmission range, and $n$ is the power loss exponent that takes a value between 2 and 4 . Each node is assigned a default maximum transmission power, Pmax to send REQUEST message. This value is included in the header of the REQUEST message. In order to adjust the transmission power, each node needs to determine the transmission power that it needs to reach each of its neighbors. When a node receives a REQUEST message from a neighbor, it simply extracts the power level with which the packet is transmitted (Pmax) and computes the required transmission power, Preq, to reach this neighbor using the following equation:

$$
\text { Preq }=P_{\text {max }} / P_{\text {recv } \mathrm{X}} \text { Pthreshold }
$$

-Equation (3)

Where Pthreshold is the minimum power for a packet to be received correctly.

\subsection{Random Broadcast Timer}

The random broadcast timer (RBT) of nodes is as follows:

$\mathrm{RBT}=[$ MaxRange - distance MaxRange $]+$

[MaxReach - unreached MaxReach]

xand ()$\times$ MaxDel

Table 1: Our Simulation parameter is as follows:

\begin{tabular}{|c|c|}
\hline Parameter & Value \\
\hline Simulator & Ns2 -2.34 \\
\hline Number of nodes & $30,50,100$ \\
\hline Simulation Time & $20 \mathrm{~min}$ \\
\hline Packet Interval & $0.01 \mathrm{sec}$ \\
\hline $\begin{array}{c}\text { Simulation } \\
\text { Landscape }\end{array}$ & $1000 \times 1000$ \\
\hline Traffic Size & CBR \\
\hline Packet Size & 1000 bytes \\
\hline Queue Length & 50 \\
\hline Initial Energy & 10 Joules \\
\hline $\begin{array}{c}\text { Node Transmission } \\
\text { range }\end{array}$ & $250 \mathrm{~m}$ \\
\hline Antenna Type & Omni directional \\
\hline Mobility Models & $\begin{array}{c}\text { Random-waypoint } \\
(0-30 \mathrm{~m} / \mathrm{s})\end{array}$ \\
\hline Routing Protocol & AODV \\
\hline MAC Protocol & IEEE 802.16 \\
\hline $\begin{array}{c}\text { Background Data } \\
\text { Traffic }\end{array}$ & CBR \\
\hline
\end{tabular}

In Equation (4) the MaxRange denotes the Maximum transmission range of a node, and MaxReach the Maximum number of neighbors that a node can cover. It is expected [5] that the transmission range and number of immediate neighbors of all nodes should not exceed 250 meters and 20 respectively, MaxRange $=250$ and MaxReach $=20$. MaxRange $=250$ is obtained by assuming $\mathrm{n}=2$ in (1) and Pthreshold $=3.652 \times 1010$ and Pmax = 0.28183815 in (2). distance denotes the distance of node $s$ from the message originating node while unreached represents the number of not-yet-covered neighbors of node $s$ (as seen by node $s$ ). rand () is a random number generator that generates a uniformly distributed number 
between $[0,1]$ and MaxDel is the pre-determined maximum rebroadcast "backoff" time.

\section{IEEE- 802.16 Mac Protocol:}

It is Fixed Broadband Wireless Access System. A reservation based medium access control (MAC) protocol has been adopted by the IEEE 802.16 standard as the basic protocol for data communication within the upstream channel. Back-off algorithm with adjustable window size resolves the collisions of request packets. It consists of the access point, BS (Base Station) and SSs(Subscriber Stations). All data traffic goes through the BS, and the BS can control the allocation of bandwidth on the radio channel. It works on Bandwidth on Demand system.

Table 2: Proposed MAC Routing Efficiency

\begin{tabular}{|c|c|c|c|}
\hline \multirow{2}{*}{$\begin{array}{l}\text { Node } \\
\text { Size }\end{array}$} & \multicolumn{3}{|c|}{ Qos parameters IEEE - 802.16 } \\
\cline { 2 - 4 } & PDR & Delay & Throughput \\
\hline 25 & 69.15 & 0.29538 & 757771.43 \\
\hline 50 & 80.04 & 0.20340 & 120032.60 \\
\hline
\end{tabular}

Main demand is to enable rapid worldwide deployment of cost-effective broadband wireless access products, and to facilitate competition in broadband access by providing alternatives to wireless broadband access. Main advantage of the protocol is fast deployment, dynamic sharing of radio resources and low cost. Multipath is negligible and it acts as the only disadvantage. Uplink / Downlink - Random access area is primarily used for the initial access but also for the signalling when the terminal has no resources allocated within the uplink phase. A standard scheduling algorithm is to be used for analysis of packet distribution.

\section{EXPERIMENTAL RESULTS}

The problem of shorter network lifetime is solved by achieving the individual energy using Adaptive configuration Algorithm. Having the survey and the collection of data from different scenario, it could be asserted that the proposed Mac protocol provides good performance and achieves quality of service better in wireless network.

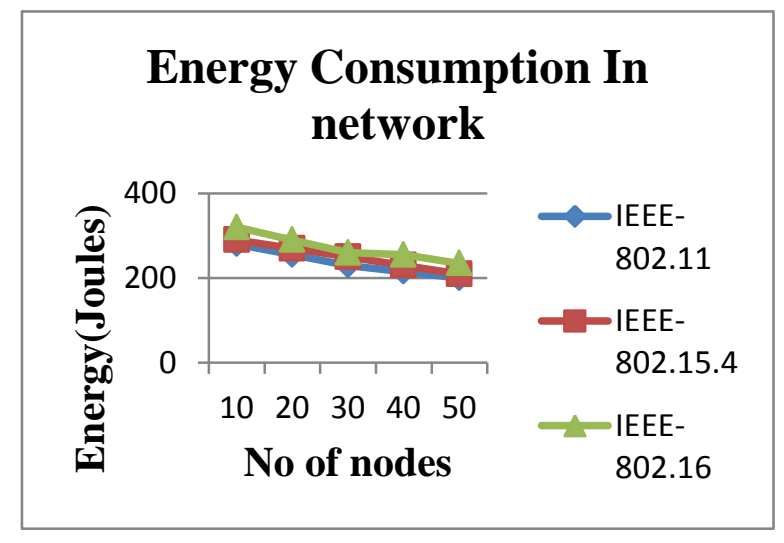

Fig 4: Energy Consumption In network

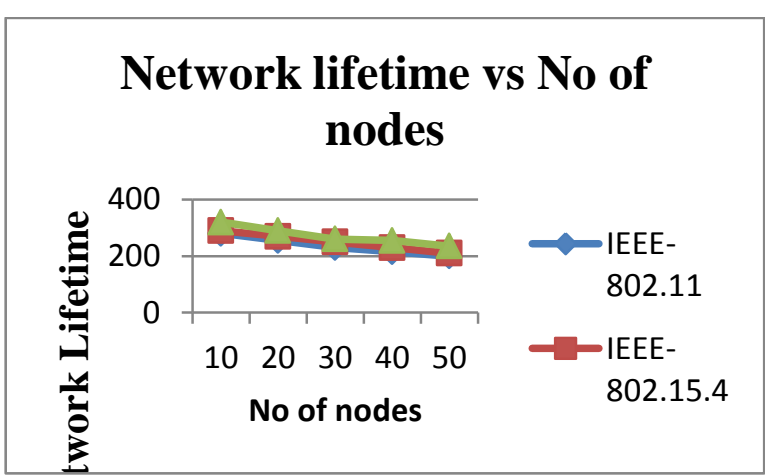

Fig 5: Network Lifetime

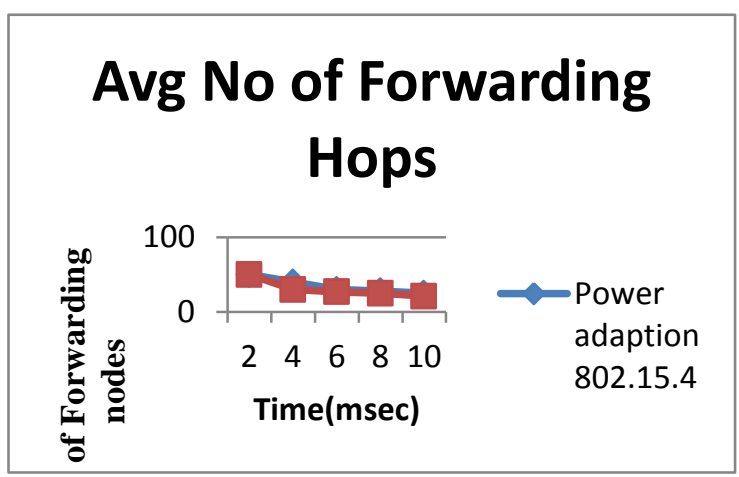

Fig 6 : Comparison of Forwarding hops

\section{Comparison of Packet Delivary Ratio}

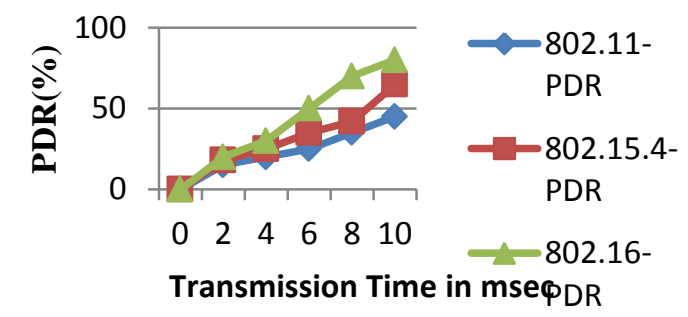

Fig 7: Comparison of Packet Delivery Ratio

\section{Comparison of Delay In Transmission}

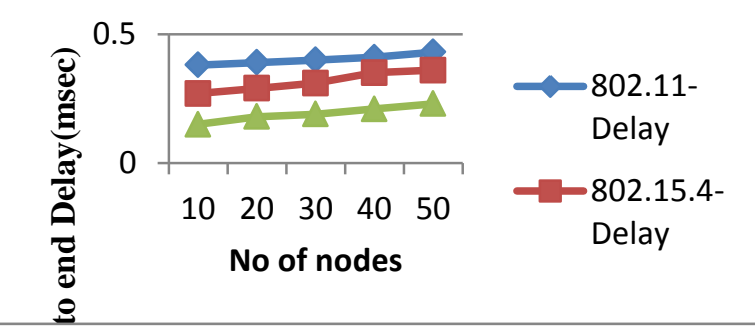

Fig 8 : Comparison of Delay In Transmission 


\section{CONCLUSION AND FUTURE WORK}

In this paper, the QOS (Quality of service) is increased using on-demand protocol which reduces the energy usage and reduces the cost of transmission; according to the individual energy of the node network lifetime will increasing. In future this work can be compared our work with enhanced Mac protocol which is having the multipath transmission as their complicity, and which randomly increases the performance in the network.

\section{REFERENCES}

[1] M. Kaynia and N. Jindal, "Performance of ALOHA and CSMA in spatially distributed wireless networks," in Proc. IEEE International Conf.on Communications (ICC), pp. 1108-1112, Beijing, China, May 2008.

[2] G. Ferrari and O. Tonguz, "MAC protocols and transport capacity in ad hoc wireless networks: Aloha versus PR-CSMA," in Proc. IEEE Military Communications Conf., Boston, USA, vol. 2, pp. 1311-1318, Oct. 2003.

[3] L. Kleinrock and F. A. Tobagi, "Packet switching in radio channels - part I: carrier sense multiple-access modes and their throughput-delay characteristics," IEEE Trans. Commun., vol. 23, pp. 1400-1416, Dec. 1975.
[4] M. Kaynia, G. E. Øien, and N. Jindal, "Impact of fading on the performance of ALOHA and CSMA," in Proc. IEEE International Workshop on Signal Processing Advances for Wireless Communications (SPAWC), pp. 394-398, June 2009.

[5] Andy An-Kai Jeng, and Rong-Hong, "Adaptive Topology Control for Mobile Ad Hoc Networks," in transcation IEEE 2011.

[6] M. S. Gokturk and O. Gurbuz, "Cooperation in wireless Ad Hoc Networks: design and performance analysis of a MAC protocol," in Proc. IEEE ICC'08, pp. 4284-4289, May 2008.

[7] B. Maham, R. Narasimhan, and A. Hjorungnes, "Energy-efficient space-time coded cooperative routing in multihop wireless networks," in Proc. IEEE GLOBECOM'09, pp. 1-7, Nov. 2009

[8] Bianchi, G.: 'Performance analysis of the IEEE 802.11 distributed coordination function', IEEE J. Sel. Areas Commun., 2000, 18, (3),pp. 535-547

[9] Bianchi, G.: 'IEEE 802.11: saturation throughput analysis', IEEE Commun. Lett., 1998, 2, (12), pp. 318-320 